\title{
SPATIAL RESOLUTION ANALYSIS OF ITERATIVE IMAGE RECONSTRUCTION WITH SEPARATE REGULARIZATION OF REAL AND IMAGINARY PARTS
}

\author{
Valur Olafsson*, Jeffrey A. Fessler \\ EECS Dept., The University of Michigan \\ \{volafsso,fessler\}@umich.edu
}

\author{
Douglas C. Noll \\ BME Dept., The University of Michigan \\ dnol1@umich.edu
}

\begin{abstract}
A common method of improving the conditioning in iterative image reconstruction is to include regularization in the reconstruction algorithm. One such regularization is the roughness penalty, which when used in the algorithm encourages smoother images. For complex valued images, the roughness penalty typically penalizes equally the real and imaginary parts. The desired resolution of the reconstructed image can then be evaluated using the local impulse response. A fast algorithm to calculate it was developed for the typical roughness penalty, used for matching the regularization parameter expediently to the desired resolution. For some cases its advantageous to penalize independently the real and imaginary parts. This paper proposes a fast algorithm to calculate the local impulse response for that penalty and applies it to an fMRI reconstruction problem.
\end{abstract}

\section{INTRODUCTION}

Iterative image reconstruction methods are growing in popularity for many imaging devices. This involves iteratively minimizing/maximizing a cost function, which fits the acquired data to a physics based signal model. Since the data received from the imaging device is usually contaminated with noise, the reconstructed image can have noise propagation due to ill-conditioning. A method to control that is to add a regularization term to the cost function. In image reconstruction, one common regularization is a roughness penalty, that encourages smoothness on the solution.

For complex valued images, the conventional roughness penalty penalizes equally the real and imaginary parts. Sometimes, unconventional roughness penalties are needed, for example separate regularization of magnitude and phase [1, 2]. Another example is separate regularization of the the real and imaginary parts, which is what this paper focuses on. This type of regularization has been used in image reconstruction in digital holography [3] and in functional MRI to jointly reconstruct images of changes in signal relaxation (real part) and off-resonance (imaginary part) [4].

${ }^{*}$ Supported in part by NIH grants R01EB002603-01 and R01DA015410-01
The weight of the roughness penalty in the cost function dictates how smooth the reconstructed image becomes. One way of choosing this weight is to choose a desired resolution for the reconstructed image. This relationship of penalty weight and resolution can be analyzed using a point spread function or impulse response function for the imaging device. For many imaging devices the impulse response is space variant, and this has led to the use of the local impulse response $[5,6]$ to analyse the spatially local resolution properties of the reconstructed image.

For spatial uniformity analysis the local impulse response must be evaluated for many pixels. To reduce compute time a fast approximate local impulse response calculation was previously derived for a quadratic penalized least-squares (QPLS) cost function using the conventional roughness penalty [6]. However, that approach is not applicable to the case of interest here, where the real and imaginary components of the image are regularized separately. This paper will present a fast method of calculating an approximate local impulse response for a separate real and imaginary roughness penalty, which is then used in the previous fMRI example [4].

\section{THEORY}

We assume the following discrete and linear model for the noisy acquired data vector $\boldsymbol{y}$ :

$$
\boldsymbol{y}=\boldsymbol{A x}+\boldsymbol{\epsilon},
$$

where $\boldsymbol{x}$ is a vector containing the unknown discrete object that is being imaged, $\boldsymbol{A}$ is a linear transform from image space to acquired data space, sometimes called the system matrix, and $\epsilon$ is a vector containing samples of the noise in the acquired data. Minimizing a QPLS cost function has been a popular method of solving the inverse problem, i.e., to estimate $\boldsymbol{x}$ from $\boldsymbol{y}$ using the model in (1). Additionally, the minimization can be solved efficiently using an iterative gradient descent algorithm, like conjugate gradient. A QPLS minimization problem can be described as follows:

$$
\begin{array}{rlrl}
(\boldsymbol{x}) & =\frac{1}{2}\|\boldsymbol{y}-\boldsymbol{A} \boldsymbol{x}\|^{2}+R(\boldsymbol{x}) \\
\widehat{\boldsymbol{x}} & = & \boldsymbol{x} & (\boldsymbol{x}),
\end{array}
$$


where $R(\cdot)$ is the penalty, used here to improve the overall conditioning of the problem and $\frac{1}{2}\|\boldsymbol{y}-\boldsymbol{A} \boldsymbol{x}\|^{2}$ is called the data fit term.

In imaging, the conventional choice for the penalty $R(\cdot)$ is the regular roughness penalty, described by:

$$
R(\boldsymbol{x})=\frac{1}{2} \beta\|\boldsymbol{C} \boldsymbol{x}\|^{2},
$$

where $C$ is a real valued matrix that evaluates the differences of neighboring pixels in $\boldsymbol{x}$ and $\beta$ is a parameter that controls how much weight the roughness penalty gets relative to the data fit term of the cost function. We would like to separately penalize the real and imaginary parts of the resulting image $\widehat{\boldsymbol{x}}$. Since the penalty in (3) does not allow for that, we propose to use the following roughness penalty:

$$
R(\boldsymbol{x})=\frac{1}{2}\left(\beta_{1}\left\|\boldsymbol{C} \boldsymbol{x}_{\mathrm{R}}\right\|^{2}+\beta_{2}\left\|\boldsymbol{C} \boldsymbol{x}_{\mathrm{I}}\right\|^{2}\right),
$$

where $\boldsymbol{x}_{\mathrm{R}}$ is the real part of $\boldsymbol{x}$ and $\boldsymbol{x}_{\mathrm{I}}$ is the imaginary part of $\boldsymbol{x}$. The parameters $\beta_{1}$ and $\beta_{2}$ control how much weight there should be on the roughness penalty of each term versus the data fit term in (2).

To control the resolution of the estimated image $\widehat{x}$, it is necessary to know the relationship of the weight of the penalty to the point spread function (PSF) of $\widehat{\boldsymbol{x}}$. It has been shown [6] for the QPLS cost function in (2) and using the roughness penalty in (3), that the local PSF or local impulse response at spatial position $n$ is given by,

$$
\boldsymbol{l}_{n}=\left(\boldsymbol{A}^{\prime} \boldsymbol{A}+\beta \boldsymbol{C}^{\prime} \boldsymbol{C}\right)^{-1} \boldsymbol{A}^{\prime} \boldsymbol{A} \boldsymbol{e}_{n}, \quad n=1, \ldots, N
$$

where $\boldsymbol{e}_{n}$ is a column vector with 1 at vector element position $n$ and zeros elsewhere (Kronecker impulse) and $\boldsymbol{l}_{n}$ is the subsequent local impulse response at spatial position $n$. However, the local impulse response in (5) does not apply when using our preferred penalty in (4), thus a new analysis is needed to find its local impulse response.

\subsection{Stacked Cost Function}

We first rewrite all the matrices and vectors in a stacked format, as follows:

$$
\begin{array}{ll}
\boldsymbol{y}_{\mathrm{S}}=\left[\begin{array}{l}
\boldsymbol{y}_{\mathrm{R}} \\
\boldsymbol{y}_{\mathrm{I}}
\end{array}\right], & \boldsymbol{x}_{\mathrm{S}}=\left[\begin{array}{c}
\boldsymbol{x}_{\mathrm{R}} \\
\boldsymbol{x}_{\mathrm{I}}
\end{array}\right], \\
\boldsymbol{A}_{\mathrm{S}}=\left[\begin{array}{cc}
\boldsymbol{A}_{\mathrm{R}} & -\boldsymbol{A}_{\mathrm{I}} \\
\boldsymbol{A}_{\mathrm{I}} & \boldsymbol{A}_{\mathrm{R}}
\end{array}\right], & \boldsymbol{C}_{\mathrm{S}}=\left[\begin{array}{cc}
\boldsymbol{C}_{1} & \mathbf{0} \\
\mathbf{0} & \boldsymbol{C}_{2}
\end{array}\right],
\end{array}
$$

where the subscripts $\mathrm{R}$ and I refer to the real and imaginary part of the variable respectively, $\boldsymbol{C}_{\mathrm{S}}$ is block diagonal with $\boldsymbol{C}_{1}=\sqrt{\beta_{1}} \boldsymbol{C}$ and $\boldsymbol{C}_{2}=\sqrt{\beta_{2}} \boldsymbol{C}$. Note that $\beta_{1}$ and $\beta_{2}$ can be chosen independently of each other. Using these definitions for the stacked matrices and vectors we can rewrite the cost function $(\boldsymbol{x})$ as an equivalent stacked cost function $\quad\left(\boldsymbol{x}_{\mathrm{S}}\right)$ such that,

$$
\left(\boldsymbol{x}_{\mathrm{S}}\right)=\frac{1}{2}\left\|\boldsymbol{y}_{\mathrm{S}}-\boldsymbol{A}_{\mathrm{S}} \boldsymbol{x}_{\mathrm{S}}\right\|^{2}+\frac{1}{2}\left\|\boldsymbol{C}_{\mathrm{S}} \boldsymbol{x}_{\mathrm{S}}\right\|^{2} .
$$

This stacked formulation of the penalty in (6) can be shown to equal (4), as follows:

$$
\begin{aligned}
\frac{1}{2}\left\|\boldsymbol{C}_{\mathrm{S}} \boldsymbol{x}_{\mathrm{S}}\right\|^{2} & =\frac{1}{2} \boldsymbol{x}_{\mathrm{S}}^{\prime} \boldsymbol{C}_{\mathrm{S}}^{\prime} \boldsymbol{C}_{\mathrm{S}} \boldsymbol{x}_{\mathrm{S}} \\
& =\frac{1}{2}\left(\beta_{1}\left\|\boldsymbol{C} \boldsymbol{x}_{\mathrm{R}}\right\|^{2}+\beta_{2}\left\|\boldsymbol{C} \boldsymbol{x}_{\mathrm{I}}\right\|^{2}\right) .
\end{aligned}
$$

As before we can now solve for the minimization problem in (6) using an iterative gradient descent algorithm such as the conjugate gradient algorithm. This can be done efficiently, but our focus here is on the spatial resolution properties of (6).

\subsection{Stacked Point Spread Function}

For the stacked cost function in (6), the stacked local point spread function can be shown to be,

$$
\boldsymbol{l}_{\mathrm{S} n}=\left(\boldsymbol{A}_{\mathrm{S}}^{\prime} \boldsymbol{A}_{\mathrm{S}}+\boldsymbol{C}_{\mathrm{S}}^{\prime} \boldsymbol{C}_{\mathrm{S}}\right)^{-1} \boldsymbol{A}_{\mathrm{S}}^{\prime} \boldsymbol{A}_{\mathrm{S}} \boldsymbol{e}_{\mathrm{S} n}
$$

with,

$$
\begin{gathered}
\boldsymbol{l}_{\mathrm{S} n}=\left[\begin{array}{c}
\boldsymbol{l}_{\mathrm{R} n} \\
\boldsymbol{l}_{\mathrm{I} n}
\end{array}\right], \quad \boldsymbol{e}_{\mathrm{S} n}=\left[\begin{array}{c}
\alpha \boldsymbol{e}_{n} \\
(1-\alpha) \boldsymbol{e}_{n}
\end{array}\right], \quad \alpha \in\{0,1\} \\
\boldsymbol{A}_{\mathrm{S}}^{\prime} \boldsymbol{A}_{\mathrm{S}}=\left[\begin{array}{cc}
\Re\left(\boldsymbol{A}^{\prime} \boldsymbol{A}\right) & -\Im\left(\boldsymbol{A}^{\prime} \boldsymbol{A}\right) \\
\Im\left(\boldsymbol{A}^{\prime} \boldsymbol{A}\right) & \Re\left(\boldsymbol{A}^{\prime} \boldsymbol{A}\right)
\end{array}\right], \\
\boldsymbol{C}_{\mathrm{S}}^{\prime} \boldsymbol{C}_{\mathrm{S}}=\left[\begin{array}{cc}
\beta_{1} \boldsymbol{C}^{\prime} \boldsymbol{C} & \mathbf{0} \\
\mathbf{0} & \beta_{2} \boldsymbol{C}^{\prime} \boldsymbol{C}
\end{array}\right],
\end{gathered}
$$

where $\alpha$ is a variable that we set to 1 to calculate the point spread function of the real part and 0 for the imaginary part. Using (8) directly to calculate the real and imaginary local impulse response would involve taking an inverse of $\boldsymbol{A}_{\mathrm{S}}^{\prime} \boldsymbol{A}_{\mathrm{S}}+$ $\boldsymbol{C}_{\mathrm{S}}^{\prime} \boldsymbol{C}_{\mathrm{S}}$ which is extremely time consuming. We would like to make the evaluation of the local impulse response fast.

\subsection{Fast Local Impulse Response Calculations}

If $\boldsymbol{A}^{\prime} \boldsymbol{A}$ and $\boldsymbol{C}^{\prime} \boldsymbol{C}$ is circulant then $\Re\left(\boldsymbol{A}^{\prime} \boldsymbol{A}\right)$ and $\Im\left(\boldsymbol{A}^{\prime} \boldsymbol{A}\right)$ are also circulant. Circulant matrices are diagonalizable using FFT transforms:

$$
\begin{gathered}
\Re\left(\boldsymbol{A}^{\prime} \boldsymbol{A}\right)=\boldsymbol{Q} \boldsymbol{\Lambda}_{1} \boldsymbol{Q}^{-1}, \quad \Im\left(\boldsymbol{A}^{\prime} \boldsymbol{A}\right)=\boldsymbol{Q} \boldsymbol{\Lambda}_{2} \boldsymbol{Q}^{-1}, \\
\boldsymbol{C}^{\prime} \boldsymbol{C}=\boldsymbol{Q} \boldsymbol{\Omega} \boldsymbol{Q}^{-1},
\end{gathered}
$$

where, $\boldsymbol{Q}^{-1}$ is the orthonormal 2D DFT matrix and $\boldsymbol{\Lambda}_{1}, \boldsymbol{\Lambda}_{2}$ and $\Omega$ are diagonal matrices given by,

$$
\begin{gathered}
\boldsymbol{\Lambda}_{1}=\quad\left\{\boldsymbol{Q}^{-1} \Re\left(\boldsymbol{A}^{\prime} \boldsymbol{A}\right) \boldsymbol{e}_{1}\right\}, \boldsymbol{\Omega}=\quad\left\{\boldsymbol{Q}^{-1} \boldsymbol{C}^{\prime} \boldsymbol{C} \boldsymbol{e}_{1}\right\}, \\
\boldsymbol{\Lambda}_{2}=\quad\left\{\boldsymbol{Q}^{-1} \Im\left(\boldsymbol{A}^{\prime} \boldsymbol{A}\right) \boldsymbol{e}_{1}\right\} .
\end{gathered}
$$


Column $n$ of a circulant matrix is a copy of its first column that has been circularly shifted $n$ places. Using this in conjunction with the circular shift property of the DFT transform, the diagonal matrices in (9) can be equivalently calculated for any $\boldsymbol{e}_{n}$, by accounting for the added phase due to the shift, as follows:

$$
\begin{aligned}
& \boldsymbol{\Lambda}_{1}=\quad\left\{\quad\left\{-i \angle\left(\boldsymbol{Q}^{-1} \boldsymbol{e}_{n}\right)\right\} \boldsymbol{Q}^{-1} \Re\left(\boldsymbol{A}^{\prime} \boldsymbol{A}\right) \boldsymbol{e}_{n}\right\}, \\
& \boldsymbol{\Lambda}_{2}=\left\{\quad\left\{-i \angle\left(\boldsymbol{Q}^{-1} \boldsymbol{e}_{n}\right)\right\} \boldsymbol{Q}^{-1} \Im\left(\boldsymbol{A}^{\prime} \boldsymbol{A}\right) \boldsymbol{e}_{n}\right\} \text {, } \\
& \boldsymbol{\Omega}=\quad\left\{\quad\left\{-i \angle\left(\boldsymbol{Q}^{-1} \boldsymbol{e}_{n}\right)\right\} \boldsymbol{Q}^{-1} \boldsymbol{C}^{\prime} \boldsymbol{C} \boldsymbol{e}_{n}\right\} .
\end{aligned}
$$

Using this FFT formulation we can rewrite (8) such that,

$$
\boldsymbol{l}_{\mathrm{S} n}=\boldsymbol{Q}_{\mathrm{D}}\left(\boldsymbol{\Lambda}_{\mathrm{S}}+\boldsymbol{\Omega}_{\mathrm{S}}\right)^{-1} \boldsymbol{\Lambda}_{\mathrm{S}} \boldsymbol{Q}_{\mathrm{D}}^{-1} \boldsymbol{e}_{\mathrm{S} n}
$$

where,

$$
\begin{gathered}
\boldsymbol{Q}_{\mathrm{D}}=\left[\begin{array}{ll}
\boldsymbol{Q} & \mathbf{0} \\
\mathbf{0} & \boldsymbol{Q}
\end{array}\right], \quad \boldsymbol{\Lambda}_{\mathrm{S}}=\left[\begin{array}{cc}
\boldsymbol{\Lambda}_{1} & -\boldsymbol{\Lambda}_{2} \\
\boldsymbol{\Lambda}_{2} & \boldsymbol{\Lambda}_{1}
\end{array}\right], \\
\boldsymbol{\Omega}_{\mathrm{S}}=\left[\begin{array}{cc}
\beta_{1} \boldsymbol{\Omega} & \mathbf{0} \\
\mathbf{0} & \beta_{2} \boldsymbol{\Omega}
\end{array}\right] .
\end{gathered}
$$

Thus for the case when $\boldsymbol{A}^{\prime} \boldsymbol{A}$ and $\boldsymbol{C}^{\prime} \boldsymbol{C}$ are circulant, we have reduced the calculation of the local impulse response considerably since it now involves only the inverse of $\boldsymbol{\Lambda}_{\mathrm{S}}+\boldsymbol{\Omega}_{\mathrm{S}}$, which is a sparse matrix of diagonal blocks, and several FFT calculations.

For many imaging problems $\boldsymbol{A}^{\prime} \boldsymbol{A}$ is not circulant, but $\boldsymbol{C}^{\prime} \boldsymbol{C}$ is. However, often we can claim that $\boldsymbol{A}^{\prime} \boldsymbol{A}$, and hence $\Re\left(\boldsymbol{A}^{\prime} \boldsymbol{A}\right)$ and $\Im\left(\boldsymbol{A}^{\prime} \boldsymbol{A}\right)$, are approximately locally circulant. This is sufficient to find the approximate local impulse response, since we assume that the local response affects only its neighboring spatial coordinates. With this in mind we can approximate $\boldsymbol{A}^{\prime} \boldsymbol{A}, \Re\left(\boldsymbol{A}^{\prime} \boldsymbol{A}\right)$ and $\Im\left(\boldsymbol{A}^{\prime} \boldsymbol{A}\right)$ locally as follows:

$$
\begin{gathered}
\boldsymbol{A}^{\prime} \boldsymbol{A} \boldsymbol{e}_{n} \approx \boldsymbol{Q} \boldsymbol{\Lambda} \boldsymbol{Q}^{-1} \boldsymbol{e}_{n}, \\
\Re\left(\boldsymbol{A}^{\prime} \boldsymbol{A}\right) \boldsymbol{e}_{n} \approx \boldsymbol{Q} \boldsymbol{\Lambda}_{1} \boldsymbol{Q}^{-1} \boldsymbol{e}_{n}, \Im\left(\boldsymbol{A}^{\prime} \boldsymbol{A}\right) \boldsymbol{e}_{n} \approx \boldsymbol{Q} \boldsymbol{\Lambda}_{2} \boldsymbol{Q}^{-1} \boldsymbol{e}_{n},
\end{gathered}
$$

where $\boldsymbol{\Lambda}_{1}$ and $\boldsymbol{\Lambda}_{2}$ are defined in (10) and $\boldsymbol{\Lambda}=\boldsymbol{\Lambda}_{1}+i \boldsymbol{\Lambda}_{2}$. Using this idea of locally circulant matrix $\boldsymbol{A}^{\prime} \boldsymbol{A}$ we can still approximate the local impulse response as in (11).

We know that $\boldsymbol{A}^{\prime} \boldsymbol{A}$ and $\boldsymbol{C}^{\prime} \boldsymbol{C}$ are nonnegative definite, and thus have real and nonnegative eigenvalues. Thus we would like the eigenvalues of $\Lambda_{\mathrm{S}}$ and $\boldsymbol{\Omega}_{\mathrm{S}}$ to be real an nonnegative. The elements of $\Omega_{\mathrm{S}}$ are nonnegative in practice due to the construction of $\boldsymbol{C}_{\mathrm{S}}$. However, special care is needed to ensure that $\boldsymbol{\Lambda}_{\mathrm{S}}$ is nonnegative, as described next.

\subsection{Fast Calculation of the Eigenvalues of $\Lambda_{S}$}

Under the approximation in (12), we have:

$$
\begin{aligned}
\boldsymbol{Q}^{-1} \Re\left(\boldsymbol{A}^{\prime} \boldsymbol{A}\right) \boldsymbol{e}_{n} & =\boldsymbol{Q}^{-1} \frac{\boldsymbol{Q} \boldsymbol{\Lambda} \boldsymbol{Q}^{-1} \boldsymbol{e}_{n}+\left(\boldsymbol{Q} \boldsymbol{\Lambda} \boldsymbol{Q}^{-1} \boldsymbol{e}_{n}\right)^{*}}{2} \\
& =\frac{1}{2} \boldsymbol{v}+\frac{1}{2} \boldsymbol{Q}^{-1}(\boldsymbol{Q} \boldsymbol{v})^{*}
\end{aligned}
$$

where $\boldsymbol{\Lambda}=\{\boldsymbol{\lambda}\}$ and $\boldsymbol{v}$ is a vector with $k$ th element $v_{k}=\lambda_{k} i \angle\left(\boldsymbol{Q}^{-1} \boldsymbol{e}_{n}\right)_{k}$ and ${ }^{*}$ is the conjugate operator. The expression above involves a conjugate of the IDFT of $\boldsymbol{v}$, which simplifies as follows:

$$
(\boldsymbol{Q} \boldsymbol{v})_{n}^{*}=\left(\sum_{k} Q_{n k} v_{k}\right)^{*}=\sum_{k} Q_{n k}^{*} v_{k}^{*}=\sum_{k} Q_{n k} v_{-k}^{*}
$$

with the index $-k \equiv-k(\quad K)$. Thus $(\boldsymbol{Q} \boldsymbol{v})^{*}$ can be rewritten as an IDFT of a circularly shifted version of $\boldsymbol{v}$. Using this in (13), we now get for the $k$ th element,

$$
\left(\boldsymbol{Q}^{-1} \Re\left(\boldsymbol{A}^{\prime} \boldsymbol{A}\right) \boldsymbol{e}_{n}\right)_{k} \approx \frac{1}{2}\left(v_{k}+v_{-k}^{*}\right) .
$$

Using this expression, the diagonal elements of $\boldsymbol{\Lambda}_{1}$ (10), which we write here as $\lambda_{1 k}$, can be expressed in terms of the eigenvalues of $\boldsymbol{A}^{\prime} \boldsymbol{A}$ as follows,

$$
\lambda_{1 k} \approx-i \angle\left(\boldsymbol{Q}^{-1} \boldsymbol{e}_{n}\right)_{k} \frac{1}{2}\left(v_{k}+v_{-k}^{*}\right)=\frac{1}{2}\left(\lambda_{k}+\lambda_{-k}\right) .
$$

This form ensures the eigenvalues of $\Re\left(\boldsymbol{A}^{\prime} \boldsymbol{A}\right)$ are approximately real and nonnegative under the local impulse response approximation. Similarly, the eigenvalues of $\Im\left(\boldsymbol{A}^{\prime} \boldsymbol{A}\right)$ under the local impulse response approximation can be written in terms of the eigenvalues of $\boldsymbol{A}^{\prime} \boldsymbol{A}$ as follows:

$$
\lambda_{2 k} \approx \frac{1}{2 i}\left(\lambda_{k}-\lambda_{-k}\right)
$$

which makes the eigenvalues of $\Im\left(\boldsymbol{A}^{\prime} \boldsymbol{A}\right)$ to be approximately purely imaginary. Using this formulation for the elements of $\boldsymbol{\Lambda}_{1}$ and $\boldsymbol{\Lambda}_{2}$ to form $\boldsymbol{\Lambda}_{\mathrm{S}}$, we can easily show that the eigenvalues of $\boldsymbol{\Lambda}_{\mathrm{S}}$ are approximately real and nonnegative, making the matrix sum $\Lambda_{\mathrm{S}}+\boldsymbol{\Omega}_{\mathrm{S}}$ in (11) positive for all values of $\beta_{1}$ and $\beta_{2}$ when evaluating the local impulse response.

Of note, we can also use these equations to form of $\boldsymbol{\Lambda}_{1}$ and $\boldsymbol{\Lambda}_{2}$, reducing the algorithm to a single pair of DFT and IDFT on $\boldsymbol{A}^{\prime} \boldsymbol{A}$. Also, the real nonnegative property might need to be enforced, since all the calculations in this subsection have all been done under the local approximation of the impulse response.

\section{SIMULATIONS}

Here we simulate an fMRI example, where we want reconstruct changes in signal relaxation $\left(\boldsymbol{x}_{\mathrm{R}}\right)$ and off-resonance $\left(\boldsymbol{x}_{\mathrm{I}}\right)$ [4]. Here, the system matrix in the signal model (1) has elements,

$$
a_{m n}=f_{n}-t_{m} z_{n} t_{m}-i 2 \pi\left(k_{m} r_{n}\right)
$$

where $f_{n}$ is the magnetization after excitation, $z_{n}$ (assumed to be known) is a complex spatial map of the initial signal relaxation (real part) and off-resonance (imaginary part) and $k_{m}$ is 


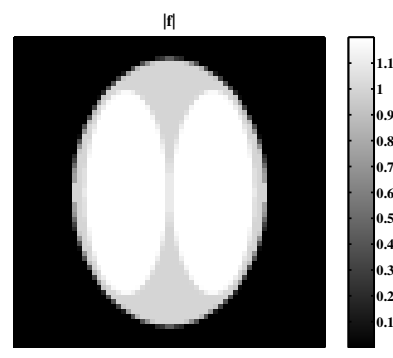

$\mathrm{z}_{1}$ (Off-Res. Map) [Hz]

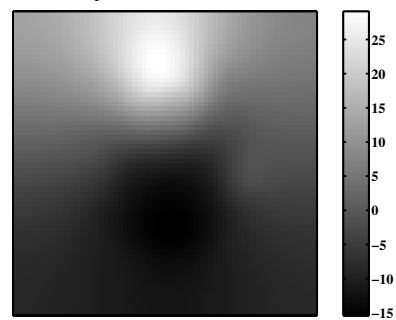

Abs FWHM Error - Real

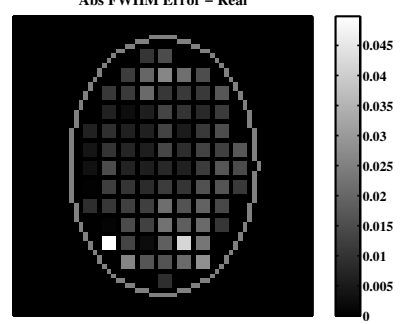

Fig. 1. Simulation maps of $|\boldsymbol{f}|$, signal relaxation $\left(z_{\mathrm{R}}\right)$ (top row) and off-resonance $\left(z_{\mathrm{I}}\right)$ (left, middle row). A map of the locations where the impulse responses were calculated (right, middle row), and a spatial map of the absolute difference in FWHM values between the exact and approximate methods, with the outline of $|\boldsymbol{f}|$ for reference (lowest row).

the k-space trajectory used to acquire the MR data, which in this case was a spiral.

In Figure 1 the simulation maps for $f_{n}$ and $z_{n}$ are shown. The exact stacked local impulse response in (8) was calculated by fully forming $\boldsymbol{A}_{\mathrm{S}}^{\prime} \boldsymbol{A}_{\mathrm{S}}$ and $\boldsymbol{C}_{\mathrm{S}}^{\prime} \boldsymbol{C}_{\mathrm{S}}$ and the approximation in (11) was calculated by forming $\Lambda_{\mathrm{S}}$ and $\boldsymbol{\Omega}_{\mathrm{S}}$ using equations from section 2.4, with $\boldsymbol{A}_{\mathrm{S}}$ formed using a fast version of the system matrix $[7,8]$.

Figure 1 shows the locations where the local impulse responses were evaluated. Since FWHM of the impulse response is used for resolution evaluation, the absolute difference in FWHM calculated using the exact and approximate stacked local impulse response is shown in Figure 1. For the exact method the average FWHM was 1.35 and 1.5 and the average error in FWHM was 0.0133 and 0.016 for the real and imaginary parts respectively. For both parts the approximation error is below $5 \%$. Notice that the maximum errors are in locations where there are edges in $f$. The exact method took $4050 \mathrm{sec}$ to run for all the locations but the fast approximate method took $32 \mathrm{sec}$, which is more then a 100-fold reduction.

\section{DISCUSSION}

We have derived a fast method to approximate the local impulse response for an iterative reconstruction algorithm, where we separately penalize the real and the imaginary parts of the image. This approximation has been shown to closely approximate the exact impulse response method with more then a 100-fold reduction in compute time. The fact that the maximum error in FWHM values occurs close to edges in $f$ indicates that local shift invariance for the impulse response does not hold well close to edges in $f$. This might improve by using a spatially variant penalty, where spatial nonuniformity in $f$ can be accounted for.

\section{REFERENCES}

[1] J. A. Fessler and D. C. Noll, "Iterative image reconstruction in MRI with separate magnitude and phase regularization," in Proc. IEEE Intl. Symp. Biomed. Imag., 2004, pp. 209-12.

[2] M. Cetin and W. C. Karl, "Feature-enhanced synthetic aperture radar image formation based on nonquadratic regularization," IEEE Trans. Im. Proc., vol. 10, no. 4, pp. 623-31, Apr. 2001.

[3] S. Sotthivirat and J. A. Fessler, "Penalized-likelihood image reconstruction for digital holography," J. Opt. Soc. Am. A, vol. 21, no. 5, pp. 737-50, May 2004.

[4] V. Olafsson, J. A. Fessler, and D. C. Noll, "Dynamic update of $R_{2}^{*}$ and field map in fMRI," in Proc. Intl. Soc. Mag. Res. Med., 2004, p. 45.

[5] D. W. Wilson and B. M. W. Tsui, "Spatial resolution properties of FB and ML-EM reconstruction methods," in Proc. IEEE Nuc. Sci. Symp. Med. Im. Conf., 1993, vol. 2, pp. 1189-93.

[6] J. A. Fessler and W. L. Rogers, "Spatial resolution properties of penalized-likelihood image reconstruction methods: Space-invariant tomographs," IEEE Trans. Im. Proc., vol. 5, no. 9, pp. 1346-58, Sept. 1996.

[7] J. A. Fessler and B. P. Sutton, "Nonuniform fast Fourier transforms using min-max interpolation," IEEE Trans. Sig. Proc., vol. 51, no. 2, pp. 560-74, Feb. 2003.

[8] B. P. Sutton, D. C. Noll, and J. A. Fessler, "Fast, iterative image reconstruction for MRI in the presence of field inhomogeneities," IEEE Trans. Med. Imag., vol. 22, no. 2, pp. 178-88, Feb. 2003. 